\title{
A Mathematical Method for Eliminating Spin Losses in Toroidal Traction Drives
}

\author{
Qingtao Li, Jie Wu, Hua Li, and Jin Yao \\ School of Manufacturing Science and Engineering, Sichuan University, Chengdu 610065, China \\ Correspondence should be addressed to Jin Yao; jinyao163@163.com
}

Received 18 September 2015; Revised 26 November 2015; Accepted 9 December 2015

Academic Editor: Roque J. Saltarén

Copyright (C) 2015 Qingtao Li et al. This is an open access article distributed under the Creative Commons Attribution License, which permits unrestricted use, distribution, and reproduction in any medium, provided the original work is properly cited.

The efficiency of the original Toroidal continuously variable transmission (CVT) is limited due to the spin losses caused by the different speed distribution in the contact area. To overcome this drawback, this paper replaces the original working surface with a new surface derived from a differential equation and proposes a novel Logarithmic CVT. Equations and ranges of the transmission ratio range, half-cone-angle, and conformity ratio, which are essential geometrical parameters of the Logarithmic CVT, are derived. A set of geometrical parameters is further recommended. With such geometrical parameters, the transmission ratio range of the Logarithmic CVT is as wide as that of the Half-Toroidal CVT. The two types of CVTs are compared with each other in terms of efficiency based on a widely accepted computational model. The results show that efficiency of the Logarithmic CVT is higher than that of Half-Toroidal CVT except for some particular situations because of the thrust bearing losses.

\section{Introduction}

Researchers have proposed various solutions for Internal Combustion (IC) engine vehicles to satisfy the increasing strict emissions standards [1]. One of these solutions is the continuously variable transmission (CVT), which is considered as an ideal drive mode because it could provide an infinite number of transmission ratios between two finite limits to maintain the IC engine operation point closer to its optimal efficiency line [2]. There are many types of CVTs which can be broadly grouped into categories such as Friction CVTs, Traction CVTs, Hydrostatic CVTs, Hydrokinetic CVTs, and Electric CVTs [3]. For IC engine vehicles, the Friction CVTs and Traction CVTs are more promising. The advantages of the Friction CVTs are simple structure, small size, and light weight. However, their torque capacities are limited, partially because they could not be easily coupled together in a series scheme [4]. To overcome this drawback, Toroidal CVTs, which belong to the Traction CVTs, have been proposed [5]. As shown in Figure 1, their main components are the input disc and roller and output disc. The power transmission of the Toroidal CVTs is achieved by shearing an extremely thin oil film generated between the roller and the disc, which can be described by the theory of Elastohydrodynamic Lubrication
(EHL) [6]. Toroidal CVTs are classified into Full-Toroidal CVTs and Half-Toroidal CVTs. Full-Toroidal CVTs, which were first proposed in 1877 [7], can transmit higher torque than that of the Friction CVTs [8]. However, their efficiency losses are up to $9 \%$ to $11 \%$ [9] because of spin losses induced by the different speed distributions in the contact area [10]. To overcome this drawback, Kraus modified the geometry and proposed the Half-Toroidal CVTs [11], which have been demonstrated to reduce the spin losses greatly and control their total efficiency losses below 5\% to 7\% [12].

So far, researchers have exerted much efforts in parameter optimizations and structural innovations to reduce the spin losses of the Toroidal CVTs. Delkhosh [13-17] and Bell [18-20] proposed a series of geometrical and tribological optimizations and gain lots of achievements; for example, it is shown that the lowest value of the spin losses occurs in low value of EHL oil temperatures and input disc rotational velocities. Akbarzadeh and Zohoor [21] proposed a sensitivity analysis to determine the share of each parameter on the mechanical efficiency. Meanwhile, a new type of double roller Full-Toroidal variator, proposed by De Novellis et al. [22], is proved to have a higher efficiency than that of the original Toroidal CVTs because it can decrease the magnitude of the spin losses. Although these studies have improved 


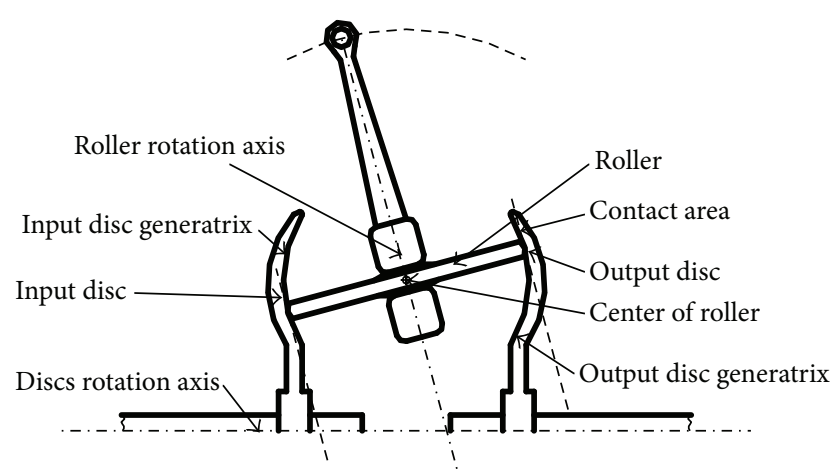

(a)

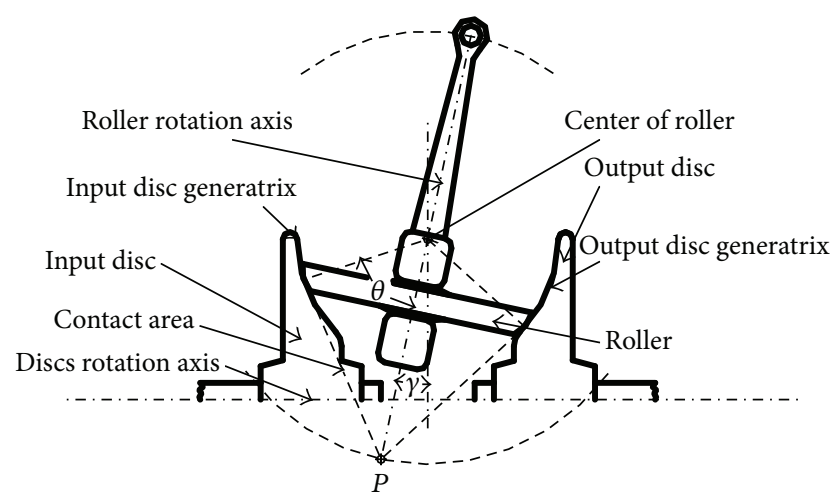

(b)

FIgure 1: The two Toroidal CVTs' geometries: (a) full-Toroidal CVT, (b) half-Toroidal CVT.

the efficiency to some extent, further improvement is difficult because the working surface of their input disc and output disc is a spherical surface which causes the spin losses that could not be totally eliminated in the full-transmission ratio range $[23,24]$. Recently, a Logarithmic CVT, which is able to eliminate the spin losses in the full-transmission ratio range, has been proposed by us [24]. The novel CVT replaces the original working surface with the new surface derived from a differential equation and has been proved to have a better performance than the Half-Toroidal CVT. However, the relationships of the geometrical parameters have not yet been discussed completely because some of the parameters were assumed to be constants and similar to that of the HalfToroidal CVTs; for example, the half-cone-angle was assumed to be $\pi / 3$ which is a familiar value in Half-Toroidal CVTs. With such geometrical parameters, the transmission ratio range of the Logarithmic CVT was considered to be narrower than that of the Half-Toroidal CVT. And the ranges of the geometrical parameters were also underestimated.

In this paper, the differential equation method is presented in detail. Equations for transmission ratio range, half-cone-angle, and conformity ratio, which are essential geometrical parameters of the proposed CVT, are derived and their relationship is also expressed in an equation. After analysis of the relationship equation, we also recommend the ranges of the parameters. A design case for the Logarithmic CVT is carried out which enables us to determine a set of the geometrical parameters. The performances of the two types of CVTs are compared with each other in terms of efficiency based on a widely accepted EHL model [25]. Interestingly, it shows that the particular shape of the disc working surface extremely reduces the spin losses in a full-transmission ratio. And the new CVT can offer a better performance than the Half-Toroidal CVT.

\section{Redesign of the Working Surface}

2.1. The Spin Losses. Efficiency losses of the Toroidal CVTs consist of bearing losses, spin losses, churning losses, and slip losses [9]. Churning losses are often neglected by researchers because of their small magnitude compared with other losses [16, 25]. As reported by Yamamoto et al. [12] and Dowson et al. [26], spin losses account for 50\%-60\% of the total losses, greater than the slip losses and bearing losses. Spin losses broadly exist in the traction transmissions; they can only be eliminated in two traction conditions [27]; that is, the parallel condition and the intersect condition. In the former condition, the rotation axes of the driving and driven elements are parallel with the contact area, which is not available for the CVTs. In the latter condition, the area and the two axes intersect at one point, which can only occur at several operation conditions of the original CVTs. The latter condition is referred to as zero-spin condition by researchers [22].

As shown in Figure 1(b), the roller rotation axis intersects with the contact areas at point $P$. According to the zerospin condition stated above, point $P$ should be located on the discs rotation axis to eliminate the spin losses. However, the working surface of the input or output disc is the spherical surface, which induces the generatrix to be a circular arc. As a result, the motion trail of the point $P$ is a circle arc which at most intersects with the discs rotation axis at two points. In other words, the spin losses can be eliminated in two special operation conditions of the Half-Toroidal CVTs, whereas, in the case of the Full-Toroidal CVT, the spin losses never vanish because the roller rotation axis is in parallel with the contact area (see Figure 1(a)).

2.2. A New Working Surface Derived from Differential Equation. To totally eliminate spin losses of the Toroidal CVTs, it is necessary to redesign the working surface of the disc. Suppose the new disc to be designed is located in a plane coordinate system $X O Y$ as shown in Figure 2, in which the $x$-axis is coinciding with the rotation axis of the disc. The function of the disc generatrix is assumed to be $y=f(x)$. $A\left(x_{0}, y_{0}\right)$ is an arbitrary point on the generatrix. Line $A P$ and line $C A$ are the common tangent line and the common normal line of the two contact curves, respectively. In other words, line $A P$ is coinciding with the projection of the contact area onto plane $X O Y . C$ is the imaginary center point of the roller. Line $C P$ is the rotation axis of the roller which intersects the line $A P$ at $P$. As the roller's generatrix is a circular arc, $P$ keeps at a fixed position relative to the roller. 


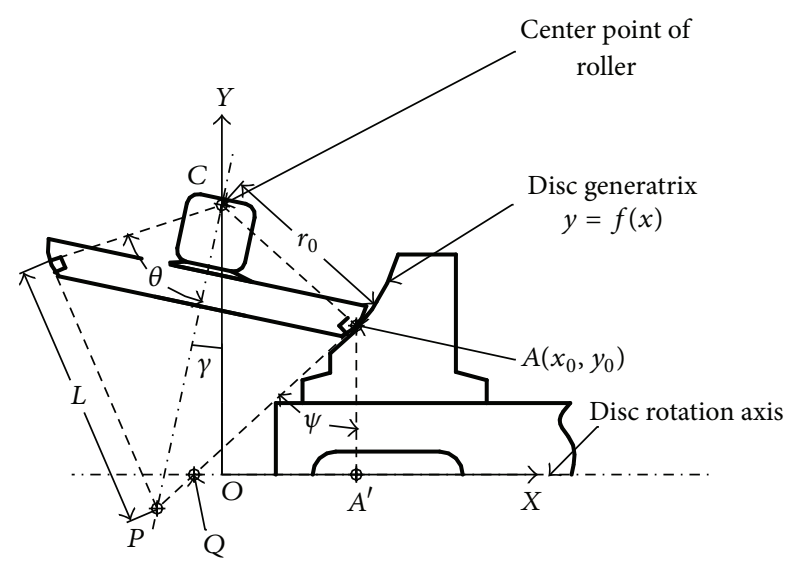

Figure 2: An ideal curve with no spin.

Line $A P$, which intersects the $x$-axis at $Q$, has a fixed length equal to $r_{0} \times \tan \theta$.

According to the zero-spin condition, $P$ must be located on the $x$-axis. But it is complicated to establish an equation group to describe such situation. Instead, the length of $A Q$ is assumed to be equal to $|A P|$. Thus, $P$ would be the intersection point of the line $C P$, the line $A P$, and the $x$ axis. And, for simplicity, the third and the fourth quadrants are ignored; thus the value of $y$ must be greater than zero in Figure 2.

The tangent equation of the curve $y=f(x)$ is

$$
f^{\prime}\left(x_{0}\right)=\frac{y-y_{0}}{x-x_{0}}
$$

The coordinate of point $Q$ is

$$
\left(x_{0}-\frac{y_{0}}{f^{\prime}\left(x_{0}\right)}, 0\right) \text {. }
$$

Thus

$$
A Q^{2}=\left(\frac{y_{0}}{f^{\prime}\left(x_{0}\right)}\right)^{2}+y_{0}^{2}
$$

Assume $|A Q|=|A P|$, and thus

$$
\begin{aligned}
A Q^{2} & =A P^{2}=L^{2}=\left(\frac{y_{0}}{f^{\prime}\left(x_{0}\right)}\right)^{2}+y_{0}{ }^{2}, \\
\left(\frac{y_{0}}{f^{\prime}\left(x_{0}\right)}\right)^{2} & =y_{0}{ }^{2}-L^{2} .
\end{aligned}
$$

Any point on the curve $y=f(x)$ should satisfy (5). After simplification, the following differential equation is derived:

$$
\frac{d y}{d x}= \pm \frac{y}{\sqrt{L^{2}-y^{2}}}
$$

Thus

$$
\begin{aligned}
\pm \int d x & =\int \frac{\sqrt{L^{2}-y^{2}}}{y} d y, \\
\pm x+C & =\sqrt{L^{2}-y^{2}}-\int y d \frac{\sqrt{L^{2}-y^{2}}}{y} \\
& =\sqrt{L^{2}-y^{2}}-\int \frac{L^{2}}{y \sqrt{L^{2}-y^{2}}} d y .
\end{aligned}
$$

Substituting $y$ with $L \sin u$ in which $u \in(0, \pi / 2$ ]

$$
\begin{aligned}
\pm x+C & =L \cos u-L \int \frac{L^{2} \cos u}{L^{2} \sin u \cos u} d u \\
& =L \cos u-L \ln (\csc u-\cot u) .
\end{aligned}
$$

The solution of (6) is

$$
\sqrt{L^{2}-y^{2}}-L \times \ln \left(\frac{y}{L-\sqrt{L^{2}-y^{2}}}\right)= \pm x+C,
$$

where $C$ is constant and $L=r_{0} \times \tan \theta$.

As shown in Figure 2, let $|C O|=h$ :

$$
\begin{aligned}
y_{0} & +r_{0} \times \frac{1}{\sqrt{1+y_{0}^{2} /\left(L^{2}-y_{0}^{2}\right)}}=y_{0}+\frac{r_{0}}{L} \sqrt{L^{2}-y_{0}^{2}} \\
& =h .
\end{aligned}
$$

As shown in Figure 2, line $A A^{\prime}$ is vertical to the disc rotation axis. $\psi$ is the angle between line $A A^{\prime}$ and line $A P$; $\psi \in(0, \pi / 2]$. It is obvious that $y \in(0, L]$. Without loss of generality, $y$ is assumed to be $L \cos \psi$. Substituting it into (10), it follows that

$$
L \cos \psi+r_{0} \sin \psi=h .
$$

Thus

$$
\sqrt{L^{2}+r_{0}^{2}} \sin \left(\psi+\arctan \frac{L}{r_{0}}\right)=h
$$

as $L=r_{0} \tan \theta$ :

$$
\sin (\psi+\theta)=\frac{h}{\sqrt{L^{2}+r_{0}^{2}}}=\sin \left(\frac{\pi}{2}-\gamma\right)
$$

in which $\theta$ and $\gamma$ are the half-cone-angle and the roller's titling angle, respectively, as shown in Figure 2. Thus, $\gamma \in[2 \theta-$ $\pi / 2, \pi / 2-\theta]$, and it follows that $\psi=(\pi / 2-\theta) \pm \gamma$. Thus, $y=L \sin (\theta \pm \gamma)$. Substituting it into (10), it follows that

$$
\begin{aligned}
& x=L \cos (\theta+\gamma)-L \ln \left[\frac{\sin (\theta+\gamma)}{1-\cos (\theta+\gamma)}\right]+C_{1}, \\
& y=L \sin (\theta+\gamma), \\
& x=-L \cos (\theta-\gamma)+L \ln \left[\frac{\sin (\theta-\gamma)}{1-\cos (\theta-\gamma)}\right]+C_{2}, \\
& y=L \sin (\theta-\gamma) .
\end{aligned}
$$






FIGURE 3: The novel CVT using the new disc generatrix.

Parametric Equation (14) describes the curve on the right and Parametric Equation (15) describes the other. $C_{1}$ and $C_{2}$ are both constants for determining the positions along the $x$-axis of the two curves.

As shown in Figure 3, such Logarithmic curves are used as the input and the output disc's generatrix, respectively, to form a novel CVT. It can be proved with mathematics that the new type of CVT could offer the no-spin traction drive in a full-transmission ratio range.

\section{Determination of the Geometrical Parameters}

3.1. Geometrical Parameters. As shown in Figure 3, $A$ is the contact point between the roller and output disc and $B$ is the contact point between the roller and the input disc. Lines $A A^{\prime}$ and $B B^{\prime}$ are vertical to the rotation axis of discs. $A^{\prime}$ and $B^{\prime}$ are their foot points. $|A P|=\left|A P^{\prime}\right|=L$ and $|C A|=|C B|=r_{0} . C^{\prime}$ is the circle center of disc generatrix.

The transmission ratio $i$ of the Logarithmic CVT is as follows:

$$
i=\frac{\left|A A^{\prime}\right|}{\left|B B^{\prime}\right|}=\frac{\sin (\theta+\gamma)}{\sin (\theta-\gamma)}
$$

where the range of $\gamma$ is temporarily assumed to be [ $2 \theta-$ $\pi / 2, \pi / 2-\theta]$, which depends on the assumption that the roller could contact with discs correctly in the whole range of $\gamma$. In other words, the relative radius of the roller needs to be shorter than the relative radii of the output and input discs at any point on the Logarithmic curve:

$$
r_{22}<r_{c}
$$

where $r_{22}$ and $r_{c}$ are the curvature radii of the roller and the logarithmic curve, respectively. It is notable that $r_{c}$ is variable along the Logarithmic curve while $r_{22}$ is a constant.
According to (14), it follows that

$$
\begin{aligned}
& \frac{d y}{d \gamma}=L \cos (\theta+\gamma) \\
& y^{\prime}=\frac{d y}{d x}=\frac{y}{\sqrt{L^{2}-y^{2}}}=\tan (\theta+\gamma) \\
& \frac{d x}{d \gamma}=L \frac{\cos ^{2}(\theta+\gamma)}{\sin (\theta+\gamma)} \\
& y^{\prime \prime}=\frac{d^{2} y}{d x^{2}}=\frac{d^{2} y}{d x d \gamma} \frac{d \gamma}{d x}=\frac{\sin (\theta+\gamma)}{L \cos ^{4}(\theta+\gamma)} .
\end{aligned}
$$

In the ranges of $\theta$ and $\gamma, \sin (\theta+\gamma)>0$ and $\cos (\theta+\gamma)>0$. Thus

$$
\begin{aligned}
\frac{1}{r_{c}} & =\frac{\left|y^{\prime \prime}\right|}{\left[1+\left(y^{\prime}\right)^{2}\right]^{3 / 2}}=\frac{\left|\sin (\theta+\gamma) / L \cos ^{4}(\theta+\gamma)\right|}{\left[1+\tan ^{2}(\theta+\gamma)\right]^{3 / 2}} \\
& =\frac{\tan (\theta+\gamma)}{r_{0} \tan \theta} .
\end{aligned}
$$

Substituting it into the inequality (17), it is obvious that the value of $r_{c}$ cannot always be greater than $r_{22}$. Thus, the range of $\gamma$ should be narrowed. Assume that $r_{22}=\delta r_{0}$, in which $\delta$ is conformity ratio of $r_{22}$ and $r_{0}$, and the range of $\gamma$ is as follows:

$$
\gamma \in\left[\theta-\arctan \left(\frac{\tan \theta}{\delta}\right), \arctan \left(\frac{\tan \theta}{\delta}\right)-\theta\right] \text {. }
$$

Substituting it into (16), the transmission ratio range $R i$, which is the ratio of the maximum to the minimum transmission ratio, is obtained as follows:

$$
R i=\frac{\sin ^{2}(\arctan (\tan \theta / \delta))}{\sin ^{2}(2 \theta-\arctan (\tan \theta / \delta))},
$$

where

$$
\frac{\tan \theta}{\delta}=\frac{r_{0} \tan \theta}{r_{22}}=\frac{L}{r_{22}}
$$

Substituting it into (21), it follows that

$$
\begin{aligned}
\sqrt{R i} & =\frac{\sin \left(\arctan \left(L / r_{22}\right)\right)}{\sin \left(2 \theta-\arctan \left(L / r_{22}\right)\right)} \\
& =\frac{L /\left|C^{\prime} P\right|}{\sin 2 \theta\left(\delta r_{0} /\left|C^{\prime} P\right|\right)-\cos 2 \theta\left(L /\left|C^{\prime} P\right|\right)} .
\end{aligned}
$$

Substituting $L=r_{0} \times \tan \theta$ into (23), we can obtain the relationship equation of the geometrical parameters:

$$
\sqrt{R i}=\frac{\tan \theta}{\delta \sin 2 \theta-\tan \theta \cos 2 \theta},
$$


where $R i, \theta$, and $\delta$ are the three parameters of the Logarithmic CVT: transmission ratio range, half-cone-angle, and conformity ratio, respectively. As (24) shows that the range of transmission ratio depends on the values of $\theta$ and $\delta$, which is a particular characteristic of the Logarithmic CVT relative to the Toroidal CVT.

The half-cone-angle $\theta$ should be in the range of $\left[40^{\circ}, 90^{\circ}\right)$. Because $\theta<90^{\circ}$ is the requirement of (9), and if $\theta<40^{\circ}$, the thrust bearing, which is on the top of the roller, will suffer an extremely large force and have a short service life. Moreover, the conformity ratio $\delta$ is assumed to be in the range of $[0.3,1)$. Because $\delta$ should be less than 1 then keep the correct point contact between the disc and roller, and if $\delta<0.3$, the contact condition will become severe. In the ranges of $\theta$ and $\delta$, the relationship of the three parameters is drawn in the graph (see Figure 4).

From Figure 4, we can see that the higher values of $\theta$ and $\delta$ cause the narrower transmission ratio range. For the Logarithmic CVT, $\delta \geq 0.8$ is unacceptable because of the extremely narrow transmission ratio range. Thus the range of $\delta$ is recommended to be $[0.3,0.8)$. By particular design, it can be inferred that the transmission ratio range of the Logarithmic CVT is as wide as that of the Toroidal CVT which is about 4.0 to 6.0 as mentioned in $[4,28-30]$. This conclusion is different from that of [24] because the latter one is under the assumption of $\theta=\pi / 3$.

3.2. Design Case. In this section, we will present a method of determining the values of the three parameters which can maintain the performance of the Logarithmic CVT similar to that of the Half-Toroidal CVT.

Firstly, determine the transmission ratio range. Due to the limitations in size and users' requirements, the transmission ratio range of the CVT is nearly $4.0-6.0$ as mentioned in Section 3.1. In this paper, it is determined to be 4.0 which is a familiar value in the Half-Toroidal CVT. Thus $R i=4$.

Secondly, determine the values of $\delta$ and $\theta$. Referring to Figure 4, it can be inferred that when $R i=4, \delta=0.5,0.4$, and 0.3 are available. However, because the low value of $\delta$ may cause a severe contact condition, $\delta=0.5$ is adopted. According to (24), we can obtain the expression for $\theta$ :

$$
\begin{aligned}
\delta \sqrt{R i} \sin 2 \theta-\sqrt{R i} \tan \theta \cos 2 \theta & =\tan \theta, \\
2 \delta \sqrt{R i} \cos ^{2} \theta-2 \sqrt{R i} \cos ^{2} \theta+\sqrt{R i} & =1, \\
\cos ^{2} \theta & =\frac{\sqrt{R i}-1}{2 \sqrt{R i}(1-\delta)} .
\end{aligned}
$$

Substituting $R i=4$ and $\delta=0.5$ into (27), $\theta=\pi / 4$.

Finally is the collision checking. The discs of the Logarithmic CVT need axial motion [24]; thus the collision checking is necessary. As shown in Figure 5, $A$ is an arbitrary point on

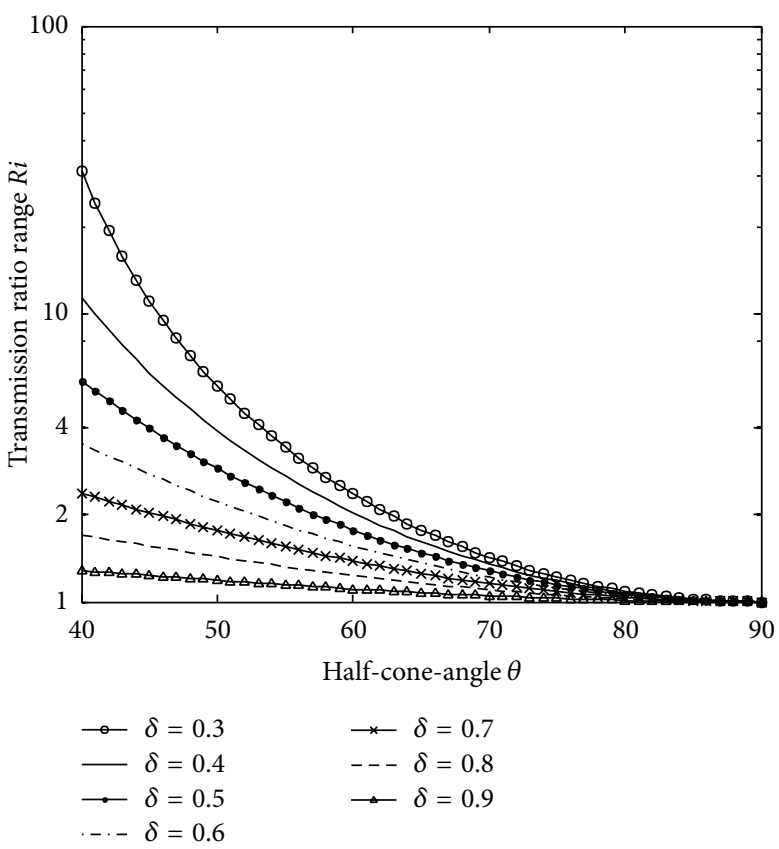

FIGURE 4: The relationship of the half-cone-angle, conformity ratio, and transmission ratio range.

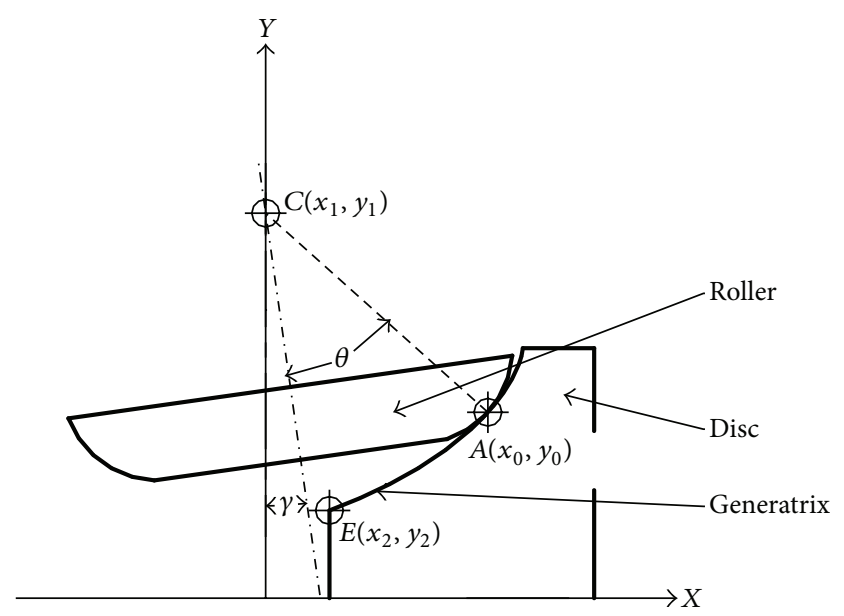

FIgURE 5: The relationship of the half-cone-angle, conformity ratio, and transmission ratio range.

the generatrix. $C$ is the imaginary center point of the roller. $E$ is the endpoint of the generatrix:

$$
x_{1}=x_{0}-r_{0} \sin (\theta+\gamma) \text {. }
$$

Substituting (14) into it, it follows that

$$
\begin{aligned}
x_{1}= & -L \ln \left(\frac{\sin (\theta+\gamma)}{1-\cos (\theta+\gamma)}\right)+C+L \cos (\theta+\gamma) \\
& -r_{0} \sin (\theta+\gamma) .
\end{aligned}
$$


Let

$$
\theta^{\prime}=\arctan \left(\frac{\tan \theta}{\delta}\right)
$$

Thus

$$
\begin{aligned}
x_{2}= & L \cos \left(2 \theta-\theta^{\prime}\right)+C \\
& -L \ln \left(\frac{\sin \left(2 \theta-\theta^{\prime}\right)}{1-\cos \left(2 \theta-\theta^{\prime}\right)}\right), \\
\frac{d x_{1}}{d \gamma}= & -L \sin (\theta+\gamma)-r_{0} \cos (\theta+\gamma) \\
& -L \frac{1-\cos (\theta+\gamma)}{\sin (\theta+\gamma)} \frac{\cos (\theta+\gamma)-1}{(1-\cos (\theta+\gamma))^{2}} \\
= & \frac{L \cos { }^{2}(\theta+\gamma)}{\sin (\theta+\gamma)}-r_{0} \cos (\theta+\gamma) \\
= & r_{0}\left(\frac{\tan \theta \cos (\theta+\gamma)}{\tan (\theta+\gamma)}-\frac{\sin (\theta+\gamma)}{\tan (\theta+\gamma)}\right) \\
= & -r_{0} \frac{\sin \gamma}{\tan (\theta+\gamma) \cos \theta} .
\end{aligned}
$$

It is obvious that when $\gamma=0, x_{1}$ is maximized. As shown in Figure 5, $C$ should be located on the left of $E$. In short, when $\gamma=0$, if

$$
x_{2}-x_{1}>0
$$

the collision would not happen. Substituting the values of $\delta$ and $\theta$ into (29), (31), and (33), we conclude that the collision cannot happen in this design case because $x_{2}-x_{1} \approx 13.29>0$.

With such adopted geometrical parameters $(R i=4, \delta=$ 0.5 , and $\theta=\pi / 4)$ and new working surfaces, a solid geometry model of the novel Logarithmic CVT is created as shown in Figure 6.

\section{Results and Discussions}

4.1. Computational Method. For the original Toroidal CVT, the calculation of the efficiency is based on the EHL theory. Because of the high pressure in the contact area, accurate calculation of the efficiency is difficult [31]. For simplicity, a series of computational methods $[4,9,25,32]$ have been proposed. However, most of these methods adopted excessive simplifications which narrows the scope of their applications. Nevertheless, the method proposed by Carbone et al. [25] has been widely accepted [17, 21, 33] because of its reasonable simplifications and acceptable precision in comparison with experimental results $[9,34]$. Therefore, this method is adopted to calculate the efficiency of the Logarithmic CVT which is similar to the Toroidal CVT.

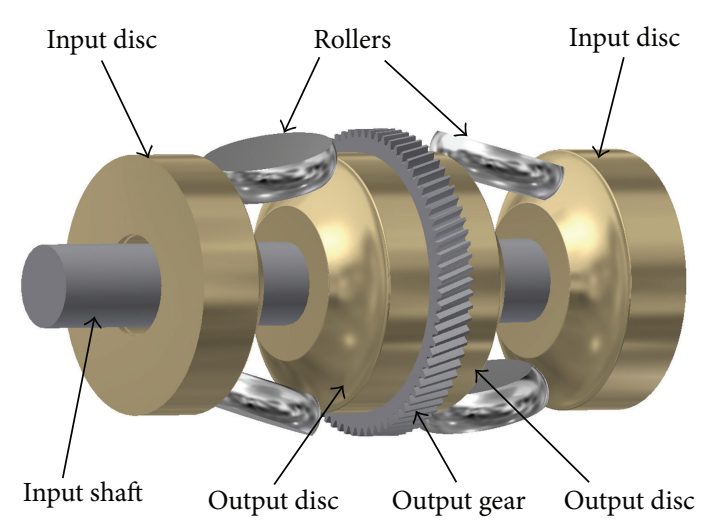

FIGURE 6: The solid geometry model of Logarithmic CVT.

Firstly the calculations of the spin ratio need to be adjusted [24]:

$$
\begin{aligned}
& \sigma_{21}=C r_{\text {in }} \sin (\theta-\gamma), \\
& \sigma_{23}=\frac{C r_{\text {out }}}{1-C r_{\text {out }}} \sin (\theta+\gamma),
\end{aligned}
$$

where $C r_{\text {in }}$ and $C r_{\text {out }}$ are the sliding coefficients of the input and output tractions, respectively. $\sigma_{21}$ and $\sigma_{23}$ are the spin ratios of the input and output tractions, respectively. From (34), it is obvious that the spin ratios of the Logarithmic CVT cannot equal zero precisely because of the sliding coefficients. However, comparing with the spin ratios of the Half-Toroidal CVT [25] shown in (35), it can be seen that the spin ratio of the Logarithmic CVT is far less than that of the Toroidal CVT, which directly promotes the efficiency:

$$
\begin{aligned}
& \sigma_{21}=\sin (\theta-\gamma)-\left(1-C r_{\text {in }}\right) \frac{1+k-\cos (\theta-\gamma)}{\tan \theta}, \\
& \sigma_{23}=\sin (\theta+\gamma)-\frac{1+k-\cos (\theta+\gamma)}{\left(1-C r_{\text {out }}\right) \tan \theta}
\end{aligned}
$$

Figure 7 shows the free body diagram of the discs and roller [25]. $F_{N}$ is the normal force at the point of contact, $F_{R}$ is the resulting load on the thrust bearing, $F_{T_{\text {in }}}$ and $F_{T_{\text {out }}}$ are the traction forces at the input and output points of contact, respectively, $T_{B L}$ is the torque resistance of the axial bearing, $T_{\text {in }}$ and $T_{\text {out }}$ are the input and output torques, respectively, $M_{S_{\text {in }}}$ and $M_{S_{\text {out }}}$ are the spin moment at the input and output points of contact, respectively, $n$ is the number of rollers per each cavity, and $m$ is the number of cavities; at last, the coordinates for the input and output contact are also defined in the diagram.

According to the force balance of the roller, the following kinetic equation of equilibrium is obtained:

$$
F_{T_{\text {in }}} r_{2}-F_{T_{\text {out }}} r_{2}-T_{B L}+M_{S_{\text {in }}} \cos \theta+M_{S_{\text {out }}} \cos \theta=0 .
$$




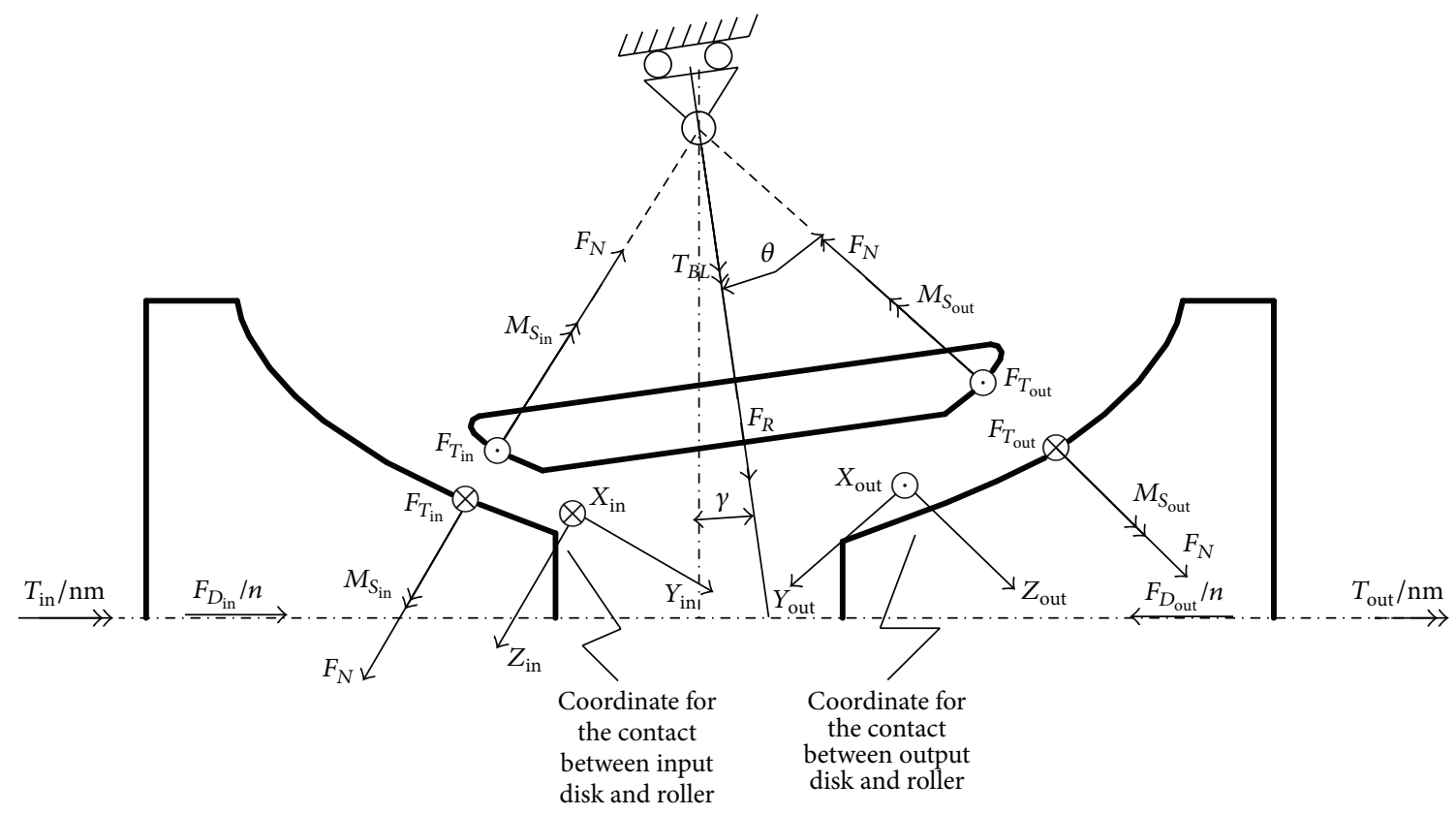

The symbol $\otimes$ is used for vector pointing far away from the reader

The symbol $\odot$ is used for vector pointing toward the reader

Figure 7: The free body diagram of the discs and roller.

$F_{T_{\text {in }}}, F_{T_{\text {out }}}, M_{S_{\text {in }}}$, and $M_{S_{\text {out }}}$ are rephrased in dimensionless forms as:

$$
\begin{aligned}
& \mu_{\text {in }}=\frac{F_{T_{\text {in }}}}{F_{N}}, \\
& \chi_{\text {in }}=\frac{M_{S_{\text {in }}}}{\left(F_{N} r_{1}\right)}, \\
& \mu_{\text {out }}=\frac{F_{T_{\text {out }}}}{F_{N}}, \\
& \chi_{\text {out }}=\frac{M_{S_{\text {out }}}}{\left(F_{N} r_{3}\right)},
\end{aligned}
$$

where $\mu_{\text {in }}$ and $\mu_{\text {out }}$ are the traction coefficients of input and output tractions, respectively, $\chi_{\text {in }}$ and $\chi_{\text {out }}$ are the spin momentum coefficients of input and output tractions, respectively. They are calculated by the numerical integration in the region of the contact area:

$$
\begin{aligned}
& \mu_{\mathrm{in}}=\tilde{a}_{X_{\mathrm{in}}} \widetilde{a}_{Y_{\mathrm{in}}} \int_{0}^{1} d R \int_{0}^{2 \pi} \tilde{\tau}_{21 X} R d \phi, \\
& \mu_{\text {out }}=-\widetilde{a}_{X_{\text {out }}} \tilde{a}_{Y_{\text {out }}} \int_{0}^{1} d R \int_{0}^{2 \pi} \tilde{\tau}_{23 X} R d \phi \text {, } \\
& \chi_{\text {in }}=\frac{\widetilde{a}_{X_{\text {in }}} \tilde{a}_{Y_{\text {in }}}}{\mathfrak{R} \widetilde{r}_{1}} \int_{0}^{1} d R \int_{0}^{2 \pi}\left(\begin{array}{c}
\widetilde{a}_{X_{\text {in }}} \widetilde{\tau}_{21 Y} \cos \phi \\
-\widetilde{a}_{Y_{\text {in }}} \widetilde{\tau}_{21 X} \sin \phi
\end{array}\right) R^{2} d \phi, \\
& \chi_{\text {out }}=\frac{\tilde{a}_{X_{\text {out }}} \tilde{a}_{Y_{\text {out }}}}{\mathfrak{R} \tilde{r}_{3}} \int_{0}^{1} d R \int_{0}^{2 \pi}\left(\begin{array}{c}
\tilde{a}_{X_{\text {out }}} \tilde{\tau}_{23 Y} \cos \phi \\
-\widetilde{a}_{Y_{\text {out }}} \tilde{\tau}_{23 X} \sin \phi
\end{array}\right) R^{2} d \phi \text {, }
\end{aligned}
$$

where $\widetilde{\tau}_{21 X}$ and $\widetilde{\tau}_{21 X}$ are the dimensionless forms of the shear stresses in $X$ and $Y$ directions at the input contact point, respectively, which can be calculated by the model proposed by Wen and Huang [31]. Similarly, $\tilde{\tau}_{23 X}$ and $\tilde{\tau}_{23 Y}$ are the dimensionless forms of the shear stresses in $X$ and $Y$ directions at the output contact point, respectively. $\mathfrak{R}$ is a calculating parameter determined by the geometrical structure of the CVT. $\widetilde{r}_{1}$ and $\widetilde{r}_{3}$ are the dimensionless forms of the parameters $\left|B B^{\prime}\right|$ and $\left|A A^{\prime}\right|$, respectively. All of them have detailed descriptions in [25].

The input and output torque coefficients $t_{\text {in }}$ and $t_{\text {out }}$ at the input and output points are

$$
\begin{aligned}
t_{\text {in }} & =\frac{T_{\text {in }} r_{0}}{m n F_{N} \widetilde{r}_{1}} ; \\
t_{\text {out }} & =\frac{T_{\text {out }} r_{0}}{m n F_{N} \tilde{r}_{3}} .
\end{aligned}
$$

Finally, the efficiency equation of the CVTs can be obtained as

$$
\nu=\left(1-C r_{\text {in }}\right)\left(1-C r_{\text {out }}\right) \frac{\mu_{\text {out }}-\chi_{\text {out }} \sin (\theta+\gamma)}{\mu_{\text {in }}-\chi_{\text {in }} \sin (\theta-\gamma)} .
$$

4.2. Comparison of the Calculated Results. Because the HalfToroidal CVT has been proved to be more efficient than the Full-Toroidal CVT, it is only necessary to compare the efficiency of the Logarithmic CVT with that of the HalfToroidal CVT in this section. The adopted geometrical and operating parameters of the two types of CVTs are shown in Table 1. To be objective, the oil type and temperature are 
TABLE 1: CVTs' geometrical and operating data.

\begin{tabular}{lcc}
\hline & $\begin{array}{c}\text { Half-Toroidal } \\
\text { CVT }\end{array}$ & $\begin{array}{c}\text { Logarithmic } \\
\text { CVT }\end{array}$ \\
\hline Cavity radius & $40 \mathrm{~mm}$ & $40 \mathrm{~mm}$ \\
Roller radius & $32 \mathrm{~mm}$ & $20 \mathrm{~mm}$ \\
Conformity ratio & 0.8 & 0.5 \\
Half-cone-angle & $\pi / 3$ & $\pi / 4$ \\
Number of cavities & 2 & 2 \\
Number of rollers' set & 2 & 2 \\
Transmission ratio range & 4 & 4 \\
\hline
\end{tabular}

TABLE 2: The fluid properties of the traction oil.

\begin{tabular}{lc}
\hline $\begin{array}{l}\text { Absolute viscosity at the atmospheric } \\
\text { pressure }\end{array}$ & $3.25 \times 10^{-3} \mathrm{~Pa} \mathrm{~s}$ \\
\hline Viscosity-pressure index & 0.85 \\
\hline Pressure-viscosity coefficient & $1.71 \times 10^{-8} \mathrm{~Pa}^{-1}$ \\
\hline $\begin{array}{l}\text { Limiting shear stress at the atmospheric } \\
\text { pressure }\end{array}$ & $0.02 \times 10^{9} \mathrm{~Pa}$ \\
\hline $\begin{array}{l}\text { Limiting shear stress constant } \\
\text { Pole pressure constant of Roelands viscosity }\end{array}$ & 0.085 \\
\hline model & $1.96 \times 10^{8} \mathrm{~Pa}$ \\
\hline Pole viscosity of Roelands viscosity model & $6.31 \times 10^{-5} \mathrm{~Pa} \mathrm{~s}$ \\
\hline
\end{tabular}

assumed to be the same, respectively. The model does not account for the influence of the temperature gradients on the fluid properties, since there is no universally accepted technique to calculate this effect [25]. The preferred methods are based on the fluid flash temperature which is $99^{\circ} \mathrm{C}$. The fluid properties of the traction oil (Santotrac 50) are shown in Table 2.

Figures 8,9 , and 10 show the efficiency of the CVTs as a function of the input traction coefficient $t_{\text {in }}$ with different transmission ratios $i$. Because the CVTs are symmetrical in geometry, the traction condition with $+\gamma$ is similar to that with $-\gamma$; for example, if $i=2$ is adopted $i=0.5$ is neglected. Therefore, three values of $i$ are adopted: $0.7,1$, and 2 .

As shown in Figure 8, it is obvious that the efficiency of the Logarithmic CVT is higher than that of the Half-Toroidal $\mathrm{CVT}$ in the whole transmission ratio range because the spin moment is eliminated theoretically. It is worth noticing that the efficiency of the Logarithmic CVT increases faster than that of the Half-Toroidal CVT, because the efficiency losses are dominated by spin losses when the input torque is low. As $t_{\text {in }}$ increases, the gap between the two curves becomes narrower, because the spin losses are more negligible in such traction condition and the half-cone-angle of the Logarithmic CVT is smaller than that of the Half-Toroidal CVT which induces larger losses of the thrust bearing. The curves in Figure 9 are similar to that in Figure 8. Whereas, the gap between the two curves becomes narrower, because the spin losses of the Half-Toroidal CVT are smaller. According to (35), when $i=2$, the spin losses of the Half-Toroidal CVT are nearly eliminated. As a result, the efficiencies of the two CVTs are nearly the same in Figure 10. Interestingly, when

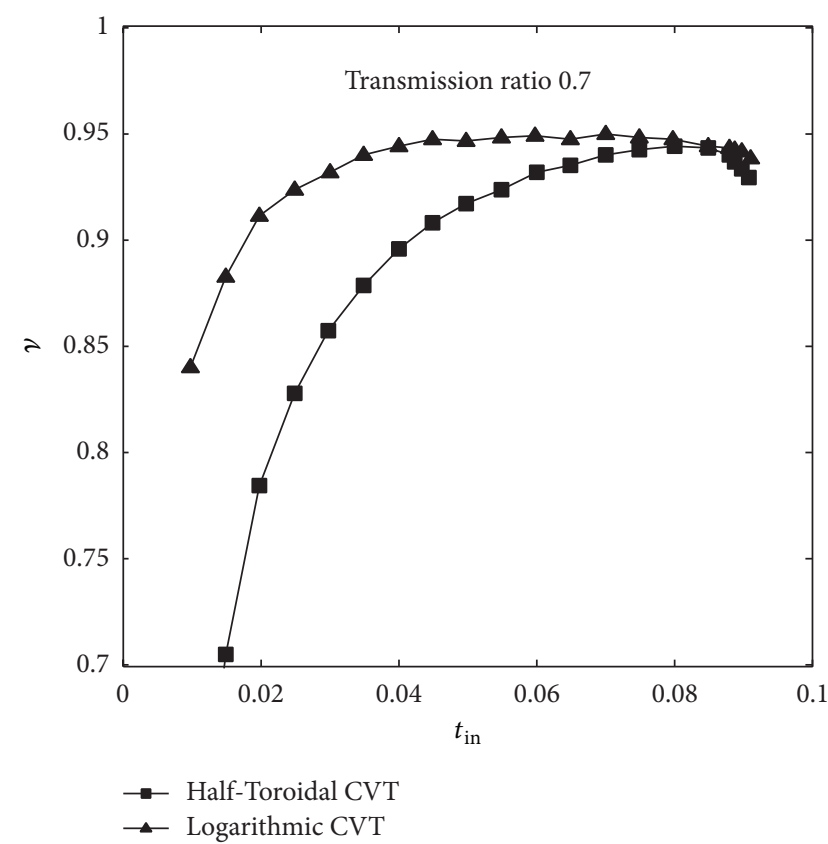

FIgURE 8: The efficiency of the CVTs as a function of the input traction coefficient $t_{\text {in }}$ with $i=0.7$.



FIGURE 9: The efficiency of the CVTs as a function of the input traction coefficient $t_{\text {in }}$ with $i=1$.

$t_{\text {in }}>0.06$ the efficiency of Logarithmic CVT is appropriately $1 \%$ lower than that of Toroidal CVT, because of the losses of the thrust bearing as stated above. In all cases we observed, as expected, that the efficiency of the Logarithmic CVT is higher than that of the Half-Toroidal CVT except for some particular situations. Specifically, when $t_{\text {in }}>0.07$, which means that the input torque is approximately higher than $336 \mathrm{Nm}$, the efficiency of the Logarithmic CVT is appropriately $1-3 \%$ 


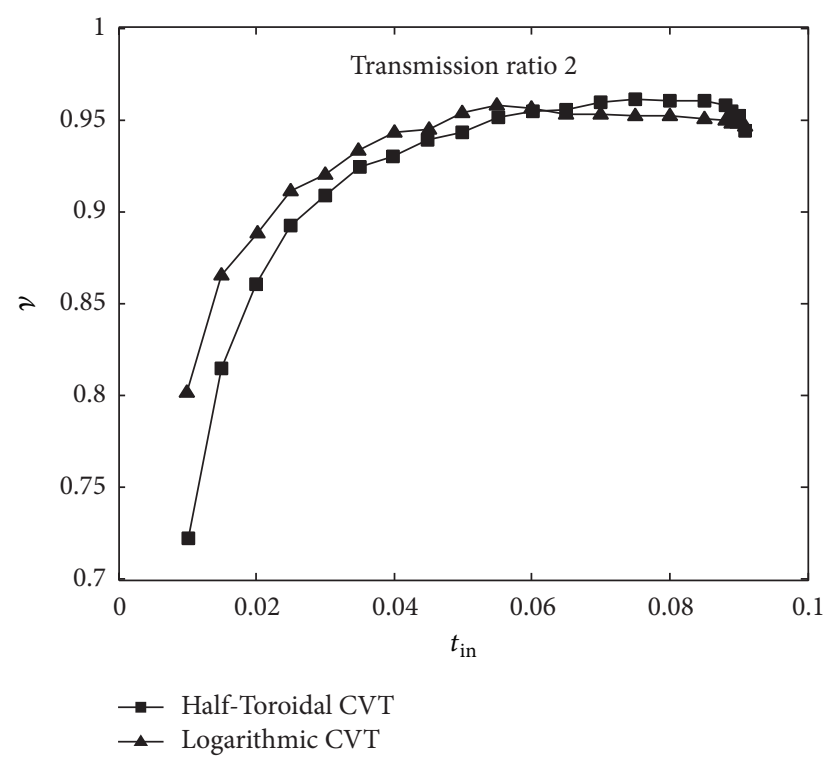

FIgure 10: The efficiency of the CVTs as a function of the input traction coefficient $t_{\text {in }}$ with $i=2$.

higher than that of the Half-Toroidal CVT, compared with $10-20 \%$ when $t_{\text {in }}<0.05$.

\section{Conclusion}

In this paper, we proposed a mathematical method for eliminating the spin losses in Toroidal traction drives. The following conclusions can be drawn:

(i) By replacing the original generatrix of the Toroidal CVT with a Logarithmic curve derived from a differential equation, the spin losses have been eliminated theoretically.

(ii) Relationship equation of transmission ratio range $R i$, half-cone-angle $\theta$, and conformity ratio $\delta$ have been derived.

(iii) The relationship of the $R i, \theta$, and $\delta$ has been drawn in graph; we also have concluded that the transmission ratio range of the Logarithmic CVT can be as wide as that of the Half-Toroidal CVT by particular design.

(iv) The ranges of $\theta$ and $\delta$ have been recommended to be $\left[40^{\circ}, 90^{\circ}\right)$ and $[0.3,0.8)$, respectively.

(v) A comparison of Logarithmic CVT with HalfToroidal CVT in terms of efficiency based on a widely accepted computational model has been carried out. The results have shown that the efficiency of the Logarithmic CVT is higher than that of the HalfToroidal CVT except for some particular situations.

\section{Conflict of Interests}

The authors declare that there is no conflict of interests regarding the publication of this paper.

\section{Acknowledgment}

This research was supported by the Applied Basic Research Programs of Sichuan Province (Grant no. 2012JY0085).

\section{References}

[1] N. Srivastava and I. Haque, "A review on belt and chain continuously variable transmissions (CVT): dynamics and control," Mechanism and Machine Theory, vol. 44, no. 1, pp. 19-41, 2009.

[2] G. Carbone, L. Mangialardi, B. Bonsen, C. Tursi, and P. A. Veenhuizen, "CVT dynamics: theory and experiments," Mechanism and Machine Theory, vol. 42, no. 4, pp. 409-428, 2007.

[3] T. Singh and S. S. Nair, "A mathematical review and comparison of continuously variable transmissions," SAE Technical Paper 922107, 1992.

[4] H. Machida, H. Itoh, T. Imanishi, and H. Tanaka, "Design principle of high power traction drive CVT," SAE Technical Paper 950675, 1995.

[5] S. Akehurst, D. A. Parker, and S. Schaaf, "CVT rolling traction drives-a review of research into their design, functionality, and modeling," Journal of Mechanical Design, vol. 128, no. 5, pp. 1165-1176, 2006.

[6] C. Brockbank and D. Burtt, "Developments in full toroidal traction drive infinitely and continuously variable transmissions," SAE Technical Paper 2007-01-3740, 2007.

[7] C. W. Hunt, "Improvement in counter-shafts for driving machinery," US Patents 197472, 1877.

[8] R. Fuchs, T. Tamura, N. Mccullough, and K. Matsumoto, "The making of the full toroidal variator," JTEKT Engineering Journal English Edition E, vol. 1006, pp. 31-36, 2009.

[9] J. P. Newall, S. Cowperthwaite, M. Hough, and A. P. Lee, "Efficiency modelling in the full toroidal variator: investigation into optimisation of EHL contact conditions to maximize contact efficiency," Tribology and Interface Engineering Series, vol. 48, pp. 245-255, 2005.

[10] X. M. Li, F. Guo, B. Fan, and P. Yang, "Influence of spinning on the rolling EHL films," Tribology International, vol. 43, no. 11, pp. 2020-2028, 2010.

[11] C. E. Kraus, "Toroidal transmission mechanism with torque loading cam means," US Patent NO.3184983, 1965.

[12] T. Yamamoto, K. Matsuda, and T. Hibi, "Analysis of the efficiency of a half-toroidal CVT," JSAE Review, vol. 22, no. 4, pp. 565-570, 2001.

[13] M. Delkhosh, M. Saadat Foumani, M. Boroushaki, M. Ekhtiari, and M. Dehghani, "Geometrical optimization of half toroidal continuously variable transmission using particle swarm optimization," Scientia Iranica, vol. 18, no. 5, pp. 1126-1132, 2011.

[14] M. Delkhosh and M. S. Foumani, "Modelling and optimization of toroidal continuously variable transmission in ECE driving cycle," International Journal of Engineering, vol. 26, no. 12, pp. 1535-1542, 2013.

[15] M. Delkhosh and M. S. Foumani, "Multi-objective geometrical optimization of full toroidal CVT,' International Journal of Automotive Technology, vol. 14, no. 5, pp. 707-715, 2013.

[16] M. Delkhosh, M. SaadatFoumani, and P. Rostami, "Application of multi-objective optimization for optimization of halftoroidal continuously variable transmission," International Journal of Engineering-Transactions C: Aspects, vol. 27, no. 9, pp. 1449-1456, 2014. 
[17] M. Delkhosh, M. S. Foumani, and M. Boroushaki, "Geometrical optimization of parallel infinitely variable transmission to decrease vehicle fuel consumption," Mechanics Based Design of Structures and Machines, vol. 42, no. 4, pp. 483-501, 2014.

[18] C. A. Bell, C. Mares, and R. Glovnea, "Multi-criteria optimisation of a continuously variable transmission," International Journal of Design Engineering, vol. 5, no. 3, pp. 232-255, 2014.

[19] C. A. Bell and R. Glovnea, "Tribological optimization of a toroidal-type continuously variable transmission," Proceedings of the Institution of Mechanical Engineers-Part J, vol. 225, no. 6, pp. 407-417, 2011.

[20] C. A. Bell, C. Mares, and R. P. Glovnea, "Concept design optimisation for continuously variable transmissions," International Journal of Mechatronics and Manufacturing Systems, vol. 4, no. 1, pp. 19-34, 2011.

[21] S. Akbarzadeh and H. Zohoor, "Sensitivity analysis of torque transmission efficiency of a half-toroidal CVT," Tech. Rep. 2006-01-1304, SAE International, 2006.

[22] L. De Novellis, G. Carbone, and L. Mangialardi, "Traction and efficiency performance of the double roller full-toroidal variator: a comparison with half-and full-toroidal drives," Journal of Mechanical Design, vol. 134, no. 7, Article ID 71005, 14 pages, 2012.

[23] Y. Zhang, X. Zhang, and W. Tobler, "A systematic model for the analysis of contact, side slip and traction of toroidal drives," Journal of Mechanical Design-Transactions of the ASME, vol. 122 , no. 4, pp. 523-528, 2000.

[24] Q. Li, H. Li, D. Yu, and J. Yao, "A novel continuously variable transmission with logarithmic disc generatrix," Mechanism and Machine Theory, vol. 93, pp. 147-162, 2015.

[25] G. Carbone, L. Mangialardi, and G. Mantriota, "A comparison of the performances of full and half toroidal traction drives," Mechanism and Machine Theory, vol. 39, no. 9, pp. 921-942, 2004.

[26] D. Dowson, C. M. Taylor, and H. Xu, "Elastohydrodynamic lubrication of elliptical contacts with spin and rolling," Proceedings of the Institution of Mechanical Engineers Part C: Journal of Mechanical Engineering Science, vol. 205, no. 3, pp. 165-174, 1991.

[27] A. A. Nabil, Performance Investigations of Half Toroidal Continuously Variable Transmissions (CVTs), Chongqing University, 2004.

[28] H. Tanaka, N. Toyoda, H. Machida, and T. Imanishi, "Development of a 6 power-roller half-toroidal CVT," NSK Technical Journal Motion and Control, vol. 9, pp. 15-26, 2000.

[29] M. Nakano, H. Kumura, J. Sugihara, H. Mori, and N. Maruyama, "Development of a large torque capacity halftoroidal CVT,' SAE Technical Paper 2000-01-0825, 2000.

[30] A. Rahman, S. B. Sharif, A. Hossain, A. K. M. Mohiuddin, and A. H. M. Z. Alam, "Kinematics and nonlinear control of an electromagnetic actuated CVT system for passenger vehicle," Journal of Mechanical Science and Technology, vol. 26, no. 7, pp. 2189-2196, 2012.

[31] S. Wen and P. Huang, Principles of Tribology, John Wiley \& Sons, 2012.

[32] T. J. Cyders, Analysis and experimental comparison of models of a new form of continuously variable transmission [Ph.D. thesis], Ohio University, 2012.

[33] S. Akehurst, D. A. Parker, and S. Schaaf, "Dynamic modeling of the milner continuously variable transmission-the basic kinematics," Journal of Mechanical Design, vol. 129, no. 11, pp. 1170-1178, 2007.
[34] J. Newall and A. Lee, "Measurement and prediction of spin losses in the EHL point contacts of the full toroidal variator," Tribology Series, vol. 43, pp. 769-779, 2003. 


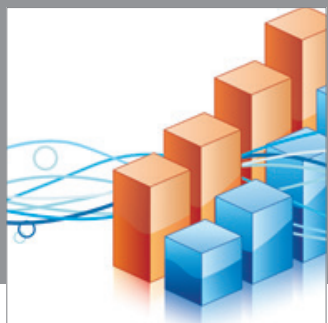

Advances in

Operations Research

mansans

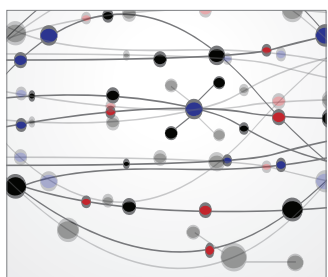

The Scientific World Journal
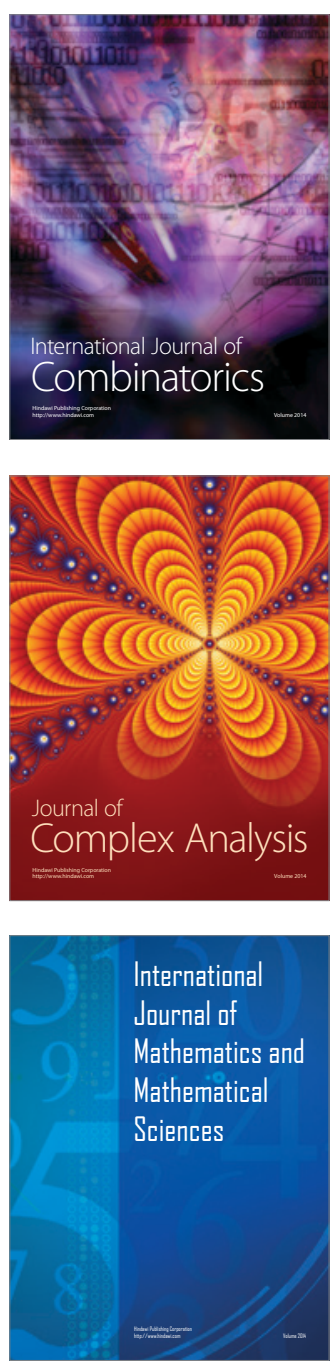
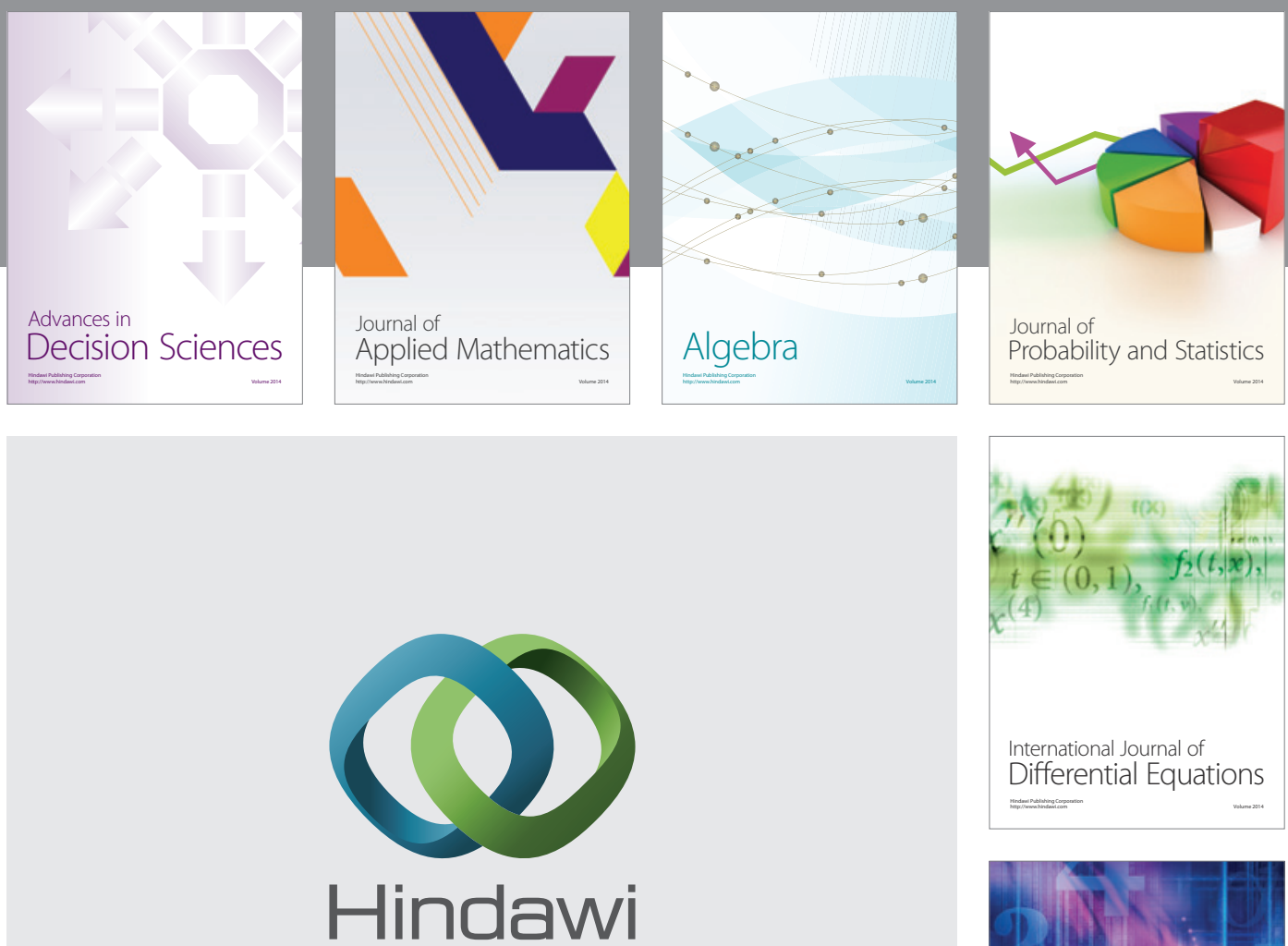

Submit your manuscripts at http://www.hindawi.com
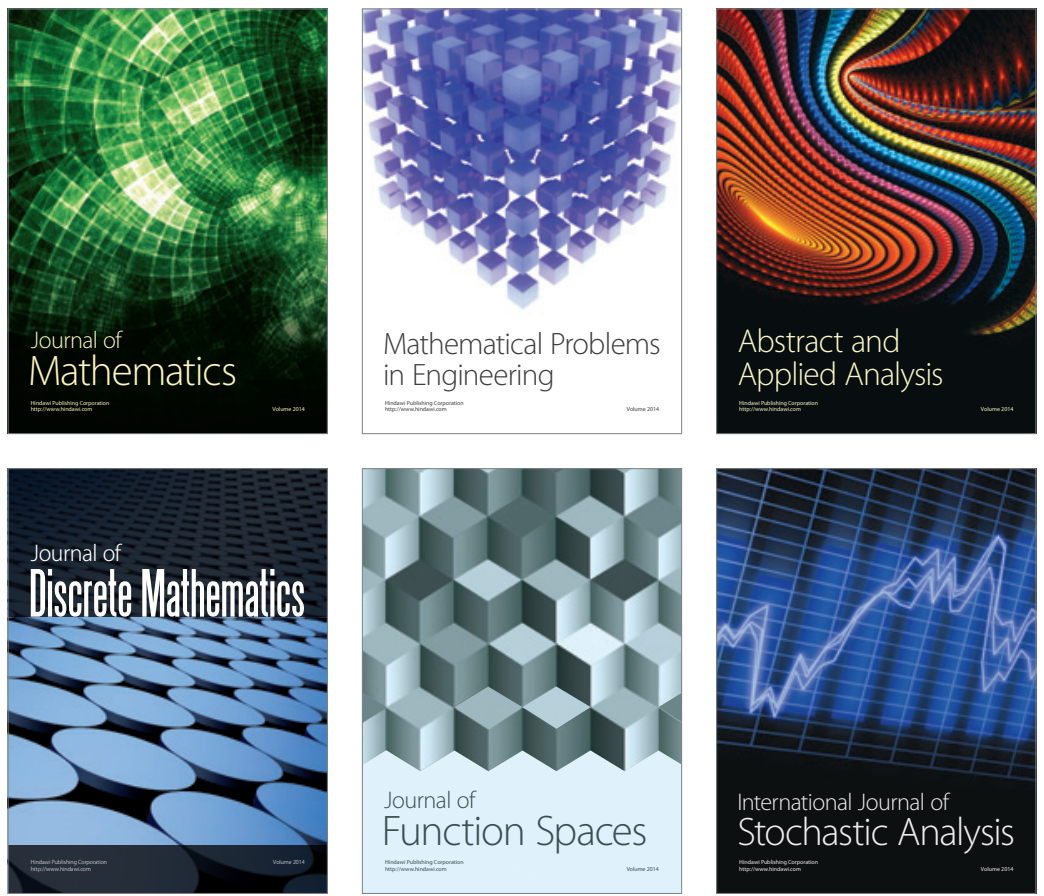

Journal of

Function Spaces

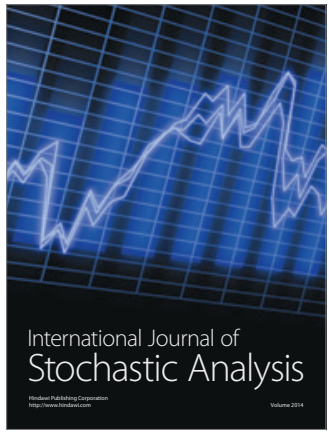

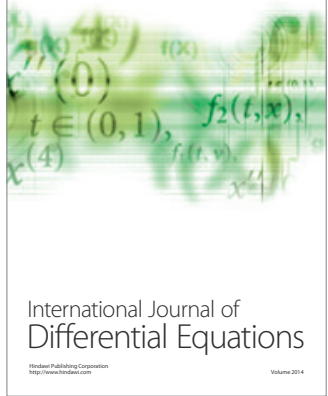
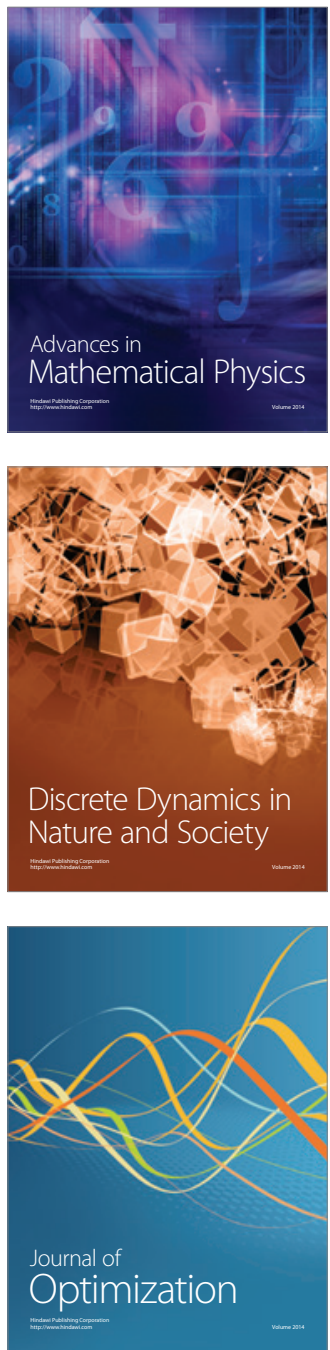